\title{
Alternative patterns of sex chromosome differentiation in Aedes aegypti (L)
}

\author{
Corey L. Campbell ${ }^{1 *}$ D, Laura B. Dickson', Saul Lozano-Fuentes ${ }^{1}$, Punita Juneja ${ }^{2}$, Francis M. Jiggins ${ }^{2}$ \\ and William C. Black IV
}

\begin{abstract}
Background: Some populations of West African Aedes aegypti, the dengue and zika vector, are reproductively incompatible; our earlier study showed that divergence and rearrangements of genes on chromosome 1, which bears the sex locus $(M)$, may be involved. We also previously described a proposed cryptic subspecies SenAae (PK10, Senegal) that had many more high inter-sex FST genes on chromosome 1 than did Ae.aegypti aegypti (Aaa, Pai Lom, Thailand). The current work more thoroughly explores the significance of those findings.
\end{abstract}

Results: Intersex standardized variance $\left(F_{S T}\right)$ of single nucleotide polymorphisms (SNPS) was characterized from genomic exome capture libraries of both sexes in representative natural populations of Aaa and SenAae. Our goal was to identify SNPs that varied in frequency between males and females, and most were expected to occur on chromosome 1. Use of the assembled AaegL4 reference alleviated the previous problem of unmapped genes. Because the $M$ locus gene nix was not captured and not present in AaegL4, the male-determining locus, per se, was not explored. Sex-associated genes were those with $F_{S T}$ values $\geq 0.100$ and/or with increased expected heterozygosity $\left(H_{\text {exp }}\right.$ one-sided T-test, $\left.p<0.05\right)$ in males. There were 85 genes common to both collections with high inter-sex $F_{S T}$ values; all genes but one were located on chromosome 1. Aaa showed the expected cluster of high inter-sex $F_{S T}$ genes proximal to the $M$ locus, whereas SenAae had inter-sex $F_{\text {ST }}$ genes along the length of chromosome 1. In addition, the Aaa M-locus proximal region showed increased $H_{\text {exp }}$ levels in males, whereas SenAae did not. In SenAae, chromosomal rearrangements and subsequent suppressed recombination may have accelerated $X-Y$ differentiation.

Conclusions: The evidence presented here is consistent with differential evolution of proto-Y chromosomes in Aaa and SenAae.

Keywords: Population genetics, Arbovirus vector, Dimorphic traits, Genomics, Evolution of reproductive proteins, Sex determination

\section{Background}

The dengue, yellow fever, chikungunya and zika vector, Aedes aegypti, has at least two major subspecies in tropical and subtropical regions; these consist principally of forest and peridomestic types [1-3]. Although morphological features such as abdominal scale patterns have been used to differentiate these groups, definitive molecular markers for subspecies identification are not yet available $[1,2,4,5]$. Population-specific differences in west African population vector competence for

\footnotetext{
* Correspondence: corey.campbell@colostate.edu

'Department of Microbiology, Immunology and Pathology, Colorado State

University, Campus Delivery 1692, Fort Collins, CO 80523, USA

Full list of author information is available at the end of the article
}

flaviviruses have been described [6,7]; and a trend toward reproductive isolation [8] may contribute toward these differences, as well as other traits $[6,7,9]$. Ae. aegypti has a dominant male-determining sex locus $(M)$ on chromosome 1, for which males are heterozygous $(\mathrm{Mm})$. This locus is primarily responsible for sex determination [10], however male and female chromosomes are also cytologically distinct [11]. The male-determining factor ( $\mathrm{M}$ factor) nix, an $\mathrm{M}$-linked myosin heavy chain gene, myo-sex, and two sex determination transcription factors have been characterized $[10,12-15]$, but little else is known about the specific genes contributing dimorphic phenotypes in aedine mosquitoes. 
Metazoan proteins involved in mating and reproduction evolve more rapidly than genes in other functional groups, and this phenomenon may contribute to reproductive isolation and subsequent speciation (reviewed in [16-18]). The opposing evolutionary forces of male sexual selection and female conflict may be involved in this process $[19,20]$. Rapid sex-associated gene evolution has been described in Anopheles mosquitoes [21] and drosophilids [22]. Haerty et al. showed rapid divergence of sex-associated genes in drosophilid males [22]. Such rapid evolution is also supported in taxa without a hemizygous X, as is the case in Ae. aegypti $[8,23]$, and has been attributed to sexual selection acting mostly on males [24]. It is expected that alleles with sexually antagonistic effects on fitness would accumulate on sex chromosomes, where they would be expressed predominantly or exclusively in the sex where they are advantageous (reviewed in [25]). In a species, such as Ae. aegypti, with recombining homomorphic sex chromosomes, these genes are expected to be enriched in regions tightly linked to the $M$ locus. Because recombination should be suppressed in the $M$ locus proximal region, differentiation of males and females likely occurs by genetic drift or possibly by selection of specific genes. For these reasons, analysis of sex-specific genetic variation in reproductively isolated mosquito populations could reveal gene diversity contributing to reproductive isolation and speciation [26].

A Senegalese sylvatic population (PK10, SenAae) has increased genetic and structural diversity at chromosome 1 compared to the type form Ae. aegypti aegypti (Aaa), possibly due to chromosomal rearrangements $[26,27]$. In addition, PK10 showed reproductive incompatibility when mated to PK10 males with different abdominal banding patterns [26]. Interestingly, this strain also lacked the expected genetic linkage of the whiteeye and the $M$ locus in $26 \%$ of genetic families [27], which was consistent with the observations of sex chromosome structural diversity. Further, high throughput sequencing (HTS) showed that overall standardized variance $\left(\mathrm{F}_{\mathrm{ST}}\right)$ was greater in SenAae than the representative type form, Aaa [27]. These unusual attributes in SenAae sex chromosomal structure and reproductive isolation led us to further explore sex-specific genomic polymorphisms in order to increase understanding of sex-specific differences in Aedes subspecies.

Therefore the over-arching goal of this study was to extend our earlier study [27] and use population genomics analyses of SenAae and Aaa to characterize sexspecific allele frequency differences. Our hypothesis was that genes with high sex-specific or inter-sex $\mathrm{F}_{\mathrm{ST}}$ values would be located proximal to the $M$ locus on chromosome 1 [28]. We used orthology information to predict whether these genes would be involved in in sex determination, reproduction and/or sexual dimorphic traits. Exome capture [29] genomic DNA (gDNA) HTS data from independent replicate pools $(n=12)$ of adult Ae.aegypti males and females were compared for two geographically and genetically distinct populations, with subsequent analysis of sex-specific single nucleotide polymorphisms (SNPs). The collections, SenAae and the type form Aaa from Thailand, have been highlighted in previous studies [26, 27, 30]. Standardized variance in SNP frequencies $\left(\mathrm{F}_{\mathrm{ST}}\right)$ was used to compare sex-specific differences [31]. Thus, in the context of this work, high inter-sex $\mathrm{F}_{\mathrm{ST}}$ values revealed SNPs that differed in frequency between males and females. We also expected that genes linked to the $M$ locus would be more heterozygous in males $[8,32]$. The Hardy-Weinberg expected heterozygosity $\left(H_{\text {exp }}\right)$ score indicated the predicted level of sex-specific genetic diversity. Genes with high $H_{\text {exp }}$ and/or $\mathrm{F}_{\mathrm{ST}}$ levels may play roles in mating, sex determination, dimorphic development or trends in reproductive isolation.

\section{Results}

Exome-wide analysis of sex-specific polymorphisms

Exome-captured HTS libraries were sequenced from pools of Aaa and SenAae males and females; Table 1 shows library-specific information and overall polymorphism statistics. Two biological replicates for each pool (12 mosquitoes per pool) of males and females from each location produced a total of eight libraries (SenAae: 2 male PK10, 2 female PK10; Aaa: 2 male Thai, 2 female Thai). Roughly 34-38 million trimmed reads were produced from SenAae, and 18-25 million reads were obtained from the Aaa collection (Additional file 1). The chromosome-length assembled Aaa genome was used as a reference for all alignments (AaegL4) [33-35]; and 90-92\% of trimmed reads aligned in each population (Additional file 1). Sexspecific polymorphisms were identified at each nucleotide site (at least 15 read counts per site) using the $\mathrm{F}_{\mathrm{ST}}$ calculation (see Methods); sex-specific $H_{\text {exp }}$ scores were also calculated [31]. SNPs that were completely fixed for both sexes but different from the reference, also known as monomorphic SNPs, were removed. The Aaa collection had about 1.9 million sex-specific polymorphisms and SenAae had about 3.0 million (Table 1). To rule out the possibility that population-specific differences arose from dissimilarities in sequencing coverage, the ratio of variant sites ( 1000) per aligned nucleotide were calculated on each chromosome (Additional file 1). In SenAae, the variant/aligned ratio ranged from 2.0-3.6 per chromosome, while in Aaa, they ranged from 2.6-4.8. Therefore, the overall relative number of variants per aligned nucleotide in Aaa was higher than that of SenAae, indicating that the features described below were not due to library size differences. 
Table 1 Polymorphisms and Coverage

\begin{tabular}{|c|c|c|c|c|}
\hline & Aaa & & SenAae & \\
\hline Monomorphic SNPs -Excluded & $21,849,618$ & & $23,861,997$ & \\
\hline Number of variant sites & $1,901,845$ & & $3,044,292$ & \\
\hline \multirow[t]{4}{*}{ Coverage per nucleotide ${ }^{a}-$} & $-\operatorname{Min}$ & 60 & & 60 \\
\hline & - Max & 3564 & & 3745 \\
\hline & -Mean & 180 & & 261.4 \\
\hline & -Median & 152 & & 214 \\
\hline \multirow[t]{2}{*}{ Allele frequency Statistics } & Aaa & & SenAae & \\
\hline & Female & Male & Female & Male \\
\hline \multicolumn{5}{|l|}{$\mathrm{H}_{\exp }$ across all genes } \\
\hline Mean $(95 \% \mathrm{cl})$ & $0.097+/-0.084$ & $0.098+/-0.084$ & $0.113+/-0.090$ & $0.112+/-0.089$ \\
\hline Median & 0.024 & 0.024 & 0.029 & 0.026 \\
\hline Mean sample variance & 0.002 & 0.002 & 0.002 & 0.002 \\
\hline Standard deviation & 0.043 & 0.043 & 0.046 & 0.046 \\
\hline \multicolumn{5}{|l|}{ Chr $1 \mathrm{H}_{\exp }$} \\
\hline Mean $(95 \% \mathrm{cl})$ & $0.099+/-0.084$ & $0.102+/-0.086$ & $0.112+/-0.089$ & $0.111+/-0.089$ \\
\hline Median & 0.025 & 0.027 & 0.031 & 0.027 \\
\hline Mean sample variance & 0.002 & 0.002 & 0.002 & 0.002 \\
\hline Standard deviation & 0.043 & 0.044 & 0.046 & 0.045 \\
\hline Increased male $H_{\text {exp }}$, T-test $p$ value & & $2.20 \mathrm{E}-16$ & & ns \\
\hline \multicolumn{5}{|l|}{ M locus proximal region } \\
\hline Mean $(95 \%$ cl) & $0.074+/-0.074$ & $0.082+/-0.078$ & $0.082+/-0.078$ & $0.081+/-0.034$ \\
\hline Median & 0.018 & 0.02 & 0.016 & 0.015 \\
\hline Mean sample variance & 0.001 & 0.002 & 0.002 & 0.0003 \\
\hline Standard deviation & 0.038 & 0.04 & 0.04 & 0.0176 \\
\hline Increased male $H_{\text {exp }}$, T-test $p$ value & & $2.20 \mathrm{E}-16$ & & ns \\
\hline
\end{tabular}

ns not significant (one-sided T test, $p<0.05$ )

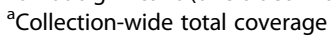

Polymorphisms were examined to identify gene-wise inter-sex $\mathrm{F}_{\mathrm{ST}}$ values (Fig. 1, Materials and Methods), which were expected to follow a beta distribution (Additional file 2). We chose a cut-off of $\mathrm{F}_{\mathrm{ST}} \geq 0.100$ to identify genes of interest for this study. This cut-off was chosen rather than a percent cut-off, such as the upper $5 \%$, because Aaa showed many fewer genes with $\mathrm{F}_{\mathrm{ST}}$ greater than 0.100 in the upper 5\% than did SenAae. For example, in the upper $5 \%$ subset, Aaa had 441 genes with $\mathrm{F}_{\mathrm{ST}}<0.100$, while SenAae had none. The chromosome-length reference allowed us to examine inter-sex $F_{\mathrm{ST}}$ averages per gene relative to each physical location. The number of high inter-sex genes was significantly higher on chromosome 1 than either chromosome 2 or 3 (Fisher's Exact test, SenAae, $p<0.0001$, Aaa, $p<0.0001)$. Interestingly, Aaa had a distinct cluster of high inter-sex $\mathrm{F}_{\mathrm{ST}}$ genes on chromosome 1, with a few in distal locations $(n=171)$. This region overlaps a similar region of high inter-sex $\mathrm{F}_{\mathrm{ST}}$ reported for the Liverpool Aaa strain [28]. This is consistent with retention of the sex locus in Aaa, with a cluster of inter-sex $\mathrm{F}_{\mathrm{ST}}$ genes proximal to nix (Fig. 1). Importantly, the male-specific $M$ locus nix was not included in the AaegL4 reference or in our capture probes $[12,13,36]$, however, predictions from AaegL4 indicate that nix is located between AAEL015064-RA and AAEL014760-RA at the location of the Aaa high $\mathrm{F}_{\mathrm{ST}}$ cluster [35].

SenAae had high inter-sex $\mathrm{F}_{\mathrm{ST}}$ genes across most of the chromosome $(n=1233)$. This pattern is consistent with extensive chromosome length $\mathrm{X}-\mathrm{Y}$ differentiation, which is different from findings of other aedine populations [28]. The high level of reported SenAae genetic diversity may have contributed to the chromosome-wide pattern [26, 27], as mosquito pools rather than individuals were evaluated in this study.

Organisms with a single sex-determining locus, such as Aedes spp., would be expected to bear sexually dimorphic heterozygosity proximal to the sex locus, and males should have greater heterozygosity at these sites. We assessed $\mathrm{H}_{\exp }$ values along the length of 


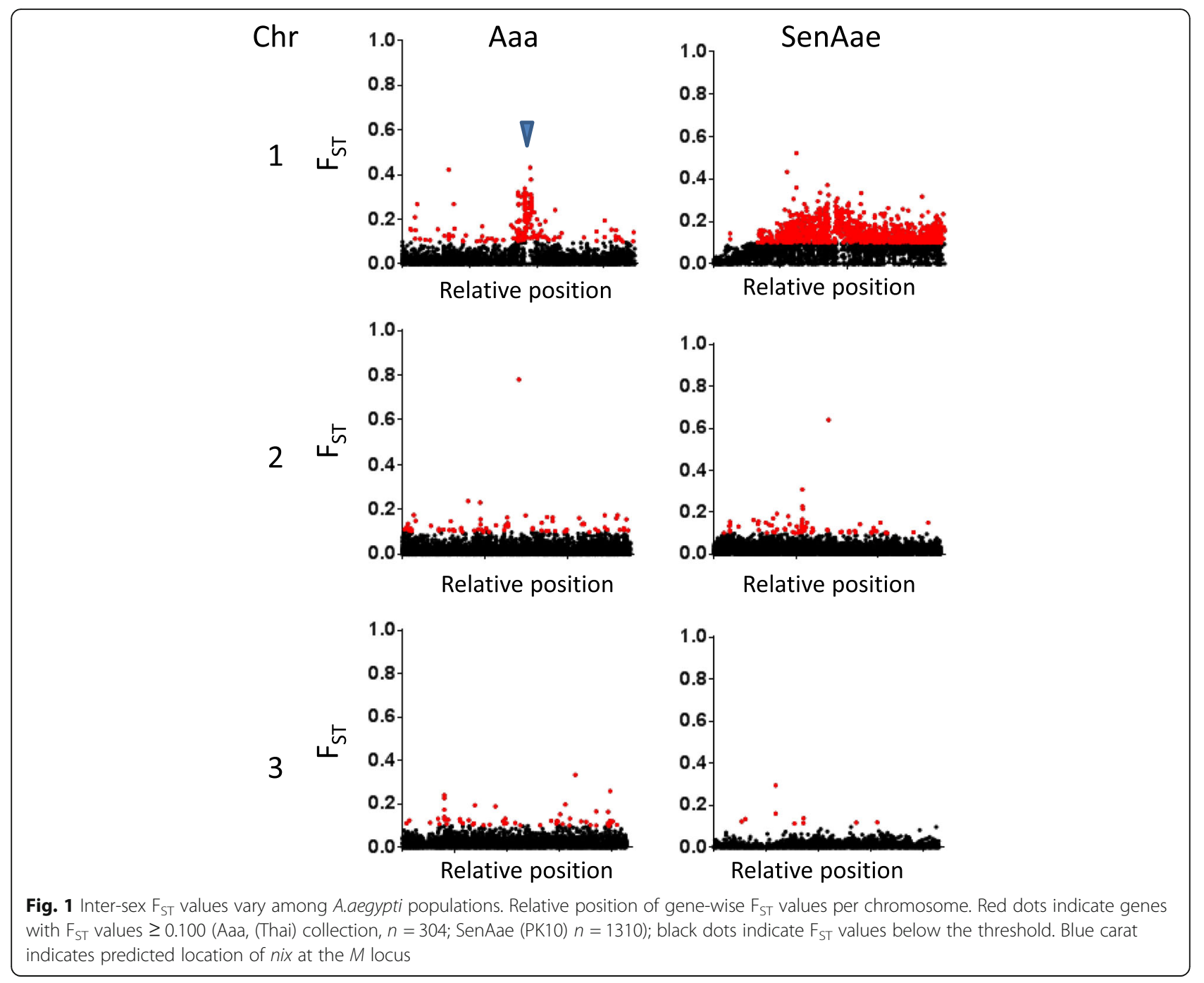

chromosome 1, testing specifically for higher average $\mathrm{H}_{\exp }$ values in males over females (one-sided T-test). Along the entire length of chromosome 1, Aaa males had increased heterozygosity levels; this was especially marked in the central third of chromosome 1 (Fig. 2 and Table 1, one-sided T-test, $p<2.2 \mathrm{E}-16$ ), which also corresponds to an area of reduced recombination reported by Fontaine et al. [28]. Curiously, increased male heterozygosity was not observed in any portion of Chromosome 1 in SenAae.

Though high inter-sex $F_{\text {ST }}$ genes were expected only on chromosome 1 , genes with association to sex were present on all three chromosomes in both populations. High inter-sex $\mathrm{F}_{\mathrm{ST}}$ genes on chromosomes 2 and 3 could be involved in processes other than sex determination, such as reproduction, sexual dimorphic development or behavior. Alternatively, this category could also include genes that contribute to sex distortion phenotypes [37-39].

We expected that $\mathrm{F}_{\mathrm{ST}}$ calculations of female-vs-female comparisons from each population should be reduced proximal to the $M$ locus. Indeed, graphs of female-vsfemale and male-vs-male comparisons indicate a marked reduction in $F_{S T}$ values proximal to the $M$ locus in females but not in males (Additional file 3). The high number of $\mathrm{F}_{\mathrm{ST}}$ values $>0.100$ shows the high level of differentiation between Aaa and SenAae.

\section{Common features of $\mathrm{X}-\mathrm{Y}$ differentiation}

Our gene-by-gene $\mathrm{F}_{\mathrm{ST}}$ calculations provided a unique opportunity to explore specific high inter-sex $\mathrm{F}_{\mathrm{ST}}$ genes that were shared among the two populations. The premise of this line of inquiry was to identify specific genes that may contribute to male-female differentiation. Indeed, a study of humans showed that high $\mathrm{F}_{\mathrm{ST}}$ genes were enriched on $\mathrm{X}$ chromosomes relative to autosomes [40]. The intersection of high inter-sex $\mathrm{F}_{\mathrm{ST}}$ genes $(\geq 0.100, n=85)$ among Aaa and SenAae was assessed (Fig. 3a, Additional file 4). As expected, chromosome 1 was most represented in this subset (Fisher's Exact test, $p<0.001$ ); just a single gene 

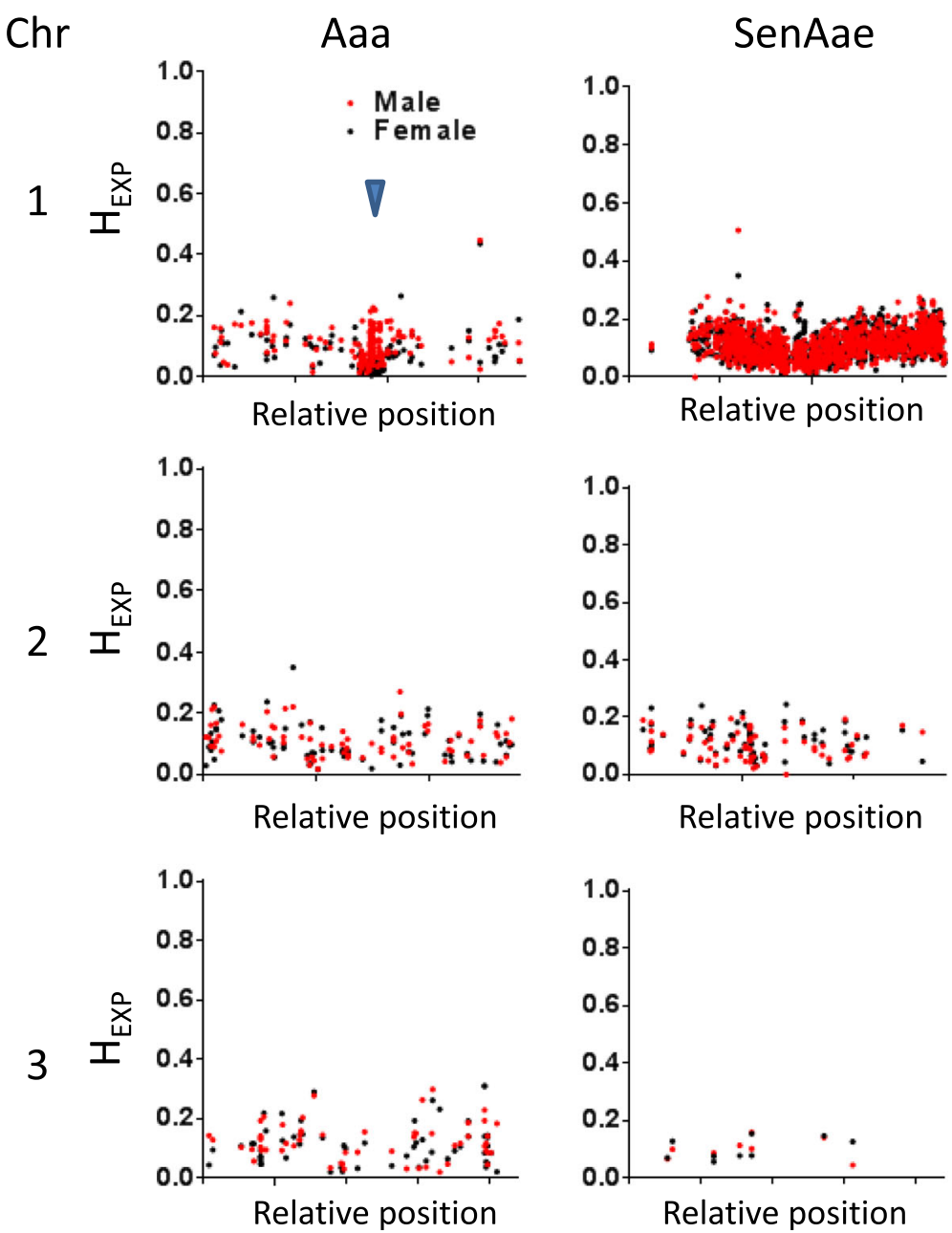

Fig. 2 Inter-sex $H_{\text {exp }}$ values vary among A.aegypti populations. Relative position of gene-wise $H_{\exp }$ values for those genes in the high $F_{S T}$ group $\left(F_{S T} \geq 0.100\right)$. Red dots indicate male gene-wise $H_{\text {exp }}$ values; black dots indicate female gene-wise $H_{\exp }$ values. Blue carat indicates predicted location of nix at the $M$ locus

(AAEL001298) on chromosome 2 was present. The data are consistent with an overall lack of common autosomal high inter-sex $\mathrm{F}_{\mathrm{ST}}$ genes, indicating that most autosomal high $\mathrm{F}_{\mathrm{ST}}$ genes are due to populationspecific trends. Importantly, high $\mathrm{F}_{\mathrm{ST}}$ values on $\mathrm{X}-\mathrm{Y}$ chromosomes could be due to genetic drift or sexspecific selection, therefore both are possible explanations for these high $\mathrm{F}_{\mathrm{ST}}$ values [41]. Nevertheless, coordinated cis-regulation of gene expression on sex chromosomes has also been described [42] and provides support for the hypothesis that sex differentiation genes, other than the $M$ locus, are present within the high $\mathrm{F}_{\mathrm{ST}}$ clusters.

To further explore the common gene set, the genes were assigned to functional categories by orthology (BLAST, $\mathrm{E}^{-20}$ cut-off) to other dipterans [43] (Fig. 3b, Additional file 4). Excluding the diverse and uncharacterized subsets, the largest subset contained genes involved in DNA repair/replication/transcription/ translation, which accounted for $18 \%$ of the total and was over-represented in this subset (hypergeometric analysis, $p<0.003)$. Nine genes had domains consistent with transcriptional activation or suppression activities. Sexlinked genes could also be those that contribute to sexually dimorphic phenotypes. For example, genes predicted to be involved in the chemosensory response (3.8\%) were also represented, though not significantly over-represented. A possible sexual dimorphic bias in chemosensory function was expected, given that males and females have distinct food sources and mating behaviors $[44,45]$.

In insects, sex determination mechanisms are highly variable across genera and species, and sometimes vary within a single species, as occurs in Musca domestica [46-48]. 


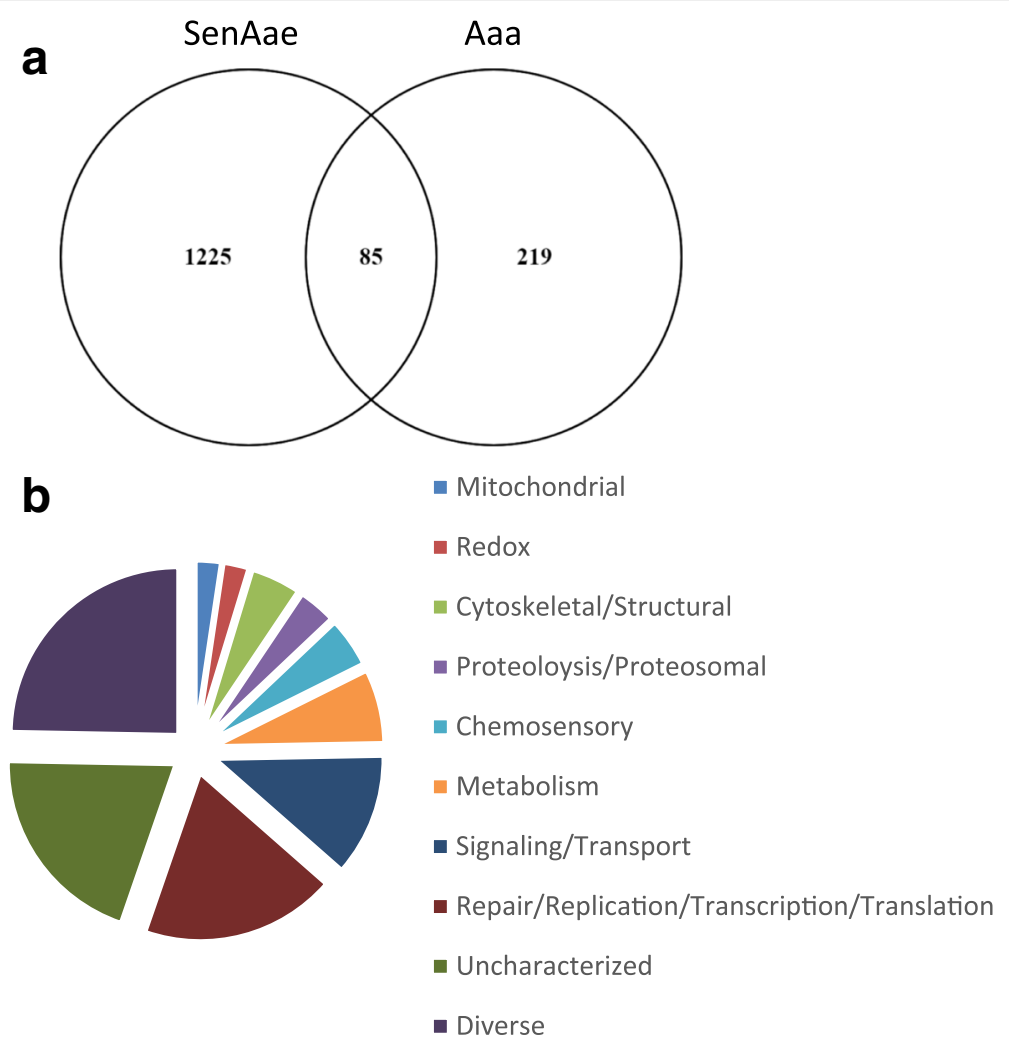

Fig. 3 Functional categories of genes showing sex-specific polymorphisms. a The intersection of genes among both populations with high inter-sex $F_{S T}$ values $(\geq 0.100)$. $\mathbf{b}$ The resulting 85 common genes were classified by functional category and are shown as a portion of the pie chart. Legend: the list of functional groups, arranged from top to bottom, is represented in the pie chart clock-wise, starting at the top-most slice

One common mechanism of dipteran sex determination, which also holds true for Ae.aegypti [14, 15], is dimorphic regulation of alternative RNA splicing mechanisms (reviewed in [49]). Just two RNA processing/splicing or sex determination genes were in the high $\mathrm{F}_{\mathrm{ST}}$ group common to both SenAae and Aaa populations, AAEL017421 and AAEL006713. Although a specific function for nucleolar protein 56 (AAEL017421) has not been identified, other nucleolar proteins are important for tissue-specific development and maintenance of heterochromatin and ribosomal RNA [50]. In addition, a U2 snRNP auxiliary factor subunit (AAEL006713) was also in this group. U-type snRNPs make up the canonical RNA-splicing machinery (reviewed in [49]).

Each collection was further interrogated to identify male seminal fluid genes [51]. 2 genes were common to both populations; they code for seminal fluid proteins AAEL010935, a gamma glutamyl transpeptidase, and AAEL014053, a vacuolar ATPase. In SenAae alone, AAEL003746, a gene with predicted 4-hydroxybutyrate CoA-transferase activity, and AAEL005790, a predicted malate dehydrogenase were identified. Also, in Aaa alone, AAEL008489, a predicted calcium ion binding protein, was identified.

\section{Discussion}

Proto-Y chromosomes evolve from autosomes upon the acquisition of a male-determining factor (reviewed in $[52,53])$. The evidence presented here is consistent with differential evolution of proto-Y chromosomes in Aaa and SenAae. We showed that Aaa had a cluster of high inter-sex $\mathrm{F}_{\mathrm{ST}}$ genes $\left(\mathrm{F}_{\mathrm{ST}}>0.100\right)$ proximal to the $M$ locus (Fig. 1). In contrast, SenAae showed high inter-sex $F_{S T}$ along the majority of chromosome 1. In Aaa but not SenAae, the $M$ locus proximal region had significantly higher male $H_{\text {exp }}$ levels (Fig. 2), which is consistent with reduced recombination. Suppressed recombination is a necessary prelude to the development of heteromorphic sex chromosomes. The reason for the absence of these features in SenAae is unknown. It could be due to a high level of genetic diversity in this population but is also consistent with accelerated X-Y differentiation. For example, chromosomal rearrangements in SenAae [26] could have contributed to accelerated X-Y differentiation of chromosome 1. Indeed, chromosomal inversions can also reduce recombination rates in proto-sex chromosomes [54].

Both groups showed population-specific trends for high inter-sex $\mathrm{F}_{\mathrm{ST}}$ genes on chromosomes 2 and 3 . The 
identification of high intersex $\mathrm{F}_{\mathrm{ST}}$ genes on autosomal chromosomes is consistent with previous studies that identified multiple independent loci contributing to the sex phenotype in other culicine species [55]. In addition, it is also consistent with the presence of high $\mathrm{F}_{\mathrm{ST}}$ autosomal genes in flies that arose from sexspecific selection due to sexual-antagonistic mechanisms [41]. Alternatively, these could also be due to sex distortion trends, though we were unable to test this hypothesis in this study. In an organism heterozygous for a given pair of alleles, we expect equal recovery of each allele in the gametes. Loci in which this fails to occur constitute "meiotic drive" (MD) or "segregation distortion" systems. Because of the ease of detection, sex ratio distortion has been the beststudied system. In Aedes aegypti, [10] the male parent determines the sex ratio in progeny and, given normal segregation, equal numbers of males and females should occur. However, departures from a 1:1 sex ratio are often observed in culicine mosquitoes and have been best studied in Aedes aegypti, wherein 35 to 45\% females are found in field collected populations [56]. A study of sex ratio in 19 laboratory strains revealed that some strains had $\sim 50 \%$ females, others had a slight excess of males ( $\sim 40 \%$ females) and a few showed distinct deviations in sex ratio (<30\% female) [57]. In 1976, a meiotic drive (MD) gene product that is tightly linked to and acts in trans with the $M$ allele was observed to cause breakage of the $m$ allele (female)-carrying chromosome [58]. It was proposed that the $m$ allele carrying chromosome is sensitive $(m s)$ or insensitive ( $m i)$ to MD. Additionally, some $m$ alleles vary in their sensitivity to distortion over a range of haplotypes [37, 59-62].

Most recently, investigators selected for a strain in which only $14.7 \%$ of progeny are females [63]. This distortion is due to an inherited factor that causes a predominance of males in certain strains and for the progeny of single pair matings. The factor is transmitted only by males [64]. Several modifiers of MD have been identified. The tolerance of Distorter locus is near the re locus at $47 \mathrm{cM}$ on chromosome 1 and results in a reduction in sex ratio distortion [39]. Another suppressor of MD is linked with the spot abdomen (s) locus on $29 \mathrm{cM}$ on chromosome 2, and an enhancer of MD was linked with the black tarsus (blt) locus at $28 \mathrm{cM}$ on chromosome 3 [65]. The actual genes associated with these genetic loci have not been identified.

\section{Conclusion}

In Aaa, increased male heterozygosity levels and high intersex $\mathrm{F}_{\mathrm{ST}}$ genes are consistent with the presence of a proto-Y chromosome (reviewed in [25]). In contrast, chromosomal rearrangements and subsequent suppressed recombination in SenAae may have accelerated
$\mathrm{X}-\mathrm{Y}$ differentiation, as the features observed in Aaa were absent. Our approach also allowed us to identify additional genes associated with sex, which may include candidates for $M$ locus modifiers. However, further characterization will be required to confirm possible mechanisms. Taken together, these data could inform transgenic strategies for vector control and the overall understanding of evolution of sex-associated genes in aedine mosquitoes.

\section{Methods}

\section{Samples and sequencing}

SenAae (PK10) and Aaa (Thai) collections were processed as follows. Deep sequencing libraries were made from pools of F1 individuals collected from the PK10 forest, Senegal in 2011 and Ae. aegypti aegypti from a collection in Pai Lom, Thailand in 2002 [7, 66, 67]. For both comparisons, mosquitoes were collected as larvae, reared to adulthood and frozen until DNA extractions. Two biological replicates for each pool (12 mosquitoes per pool) of males and females from each location produced a total of eight libraries (2 male PK10, 2 female PK10, 2 male Thai, 2 female Thai). Prior to pooling, DNA in individual mosquitoes was quantified using Pico Green (Life Technologies, Thermo Fisher Scientific Inc.) and equal amounts of DNA per mosquito were pooled. A Covaris S2 sonicator (Covaris Ltd., Brighton UK) sheared pooled DNA to an average size of $500 \mathrm{bp}$. Sonication conditions were: duty cycle $10 \%$, Intensity 5.0 , Cycles per burst 200, Duration 40 s, Mode Frequency sweeping, Displayed Power $23 \mathrm{~W}$, Temperature $5.5^{\circ}$ to $6{ }^{\circ} \mathrm{C}$. Each TruSeq DNA LT (v.2) library was prepared using $1 \mu \mathrm{g}$ of sheared genomic DNA following manufacturer's recommendations. Equimolar quantities of prepared libraries were pooled and enriched for coding sequences by exome capture using custom SeqCap EZ Developer probes (Nimblegen) [29]. In total, 26.7 Mb of the genome (2\%) was targeted for enrichment, as described elsewhere [29]. Overlapping probes covering the protein coding sequence (not including UTRs) in the AaegL1.3 gene annotations (https://www.vectorbase.org/organisms/ aedes-aegypti/liverpool-lvp/AaegL1.3) were produced by Nimblegen. Enrichment followed the Nimblegen SeqCap EZ protocol. Briefly, pooled TruSeq libraries were hybridized to the probes for $64 \mathrm{~h}$, unbound DNA was washed away, and the targeted DNA was eluted and amplified. These were then sequenced on 2 lanes of a HiSeq2000 (Illumina) for paired-end $2 \times 100$ nt sequencing. TruSeq library preparation, exome capture and sequencing were performed by the High-Throughput Genomics Group at the Wellcome Trust Centre for Human Genetics (Oxford, UK) and produced reads with quality scores $>30$. 


\section{Bioinformatics}

\section{Alignments and population genetics pipeline}

All raw reads were trimmed of adapters and filtered using cutadapt (v. 1.14) [68]. The AaegL4 genome build [35] of 18,769 transcripts was used, including all 5'UTRs, exons, introns, 3'UTRs. The 5' and 3' non-transcribed regions in previously reported alignments were excluded [27]. Individual replicate fastq files were aligned to the AaegL4 genome using GSNAP (version 2017-02-25), allowing 10\% divergence [69]. Using SAMtools "mpileup" command [70], GSNAP outputs were converted to *.mpileup files. The "readcounts" command in Varscan2 (v2.3.5) [71] was used to convert *.mpileup files to readcounts output, using the following options: -min-coverage 15 -min-base-qual 30. The readcounts output listed each SNP as a single row and A, C, G, T, in/del in columns.

To address possible sequencing errors, the following steps were taken: 1) a minimum of 15 variants per SNP site were required for a site to be considered; 2) only reads with Q30 passed trimming (cutadapt); this quality score was also required at each base upon alignment to the reference; 3) only those SNP sites that were present in both replicate libraries were included in $\mathrm{F}_{\mathrm{ST}}$ calculations. PCR duplicates were not removed, because of the evidence that removal does not significantly alter variant calls [72].

For each SNP, in-house FORTRAN (F77) scripts (available on request) used the variant coverage per SNP site to calculate the Fumagalli $\mathrm{F}_{\mathrm{ST}}$. Between-group component $\left(a_{s}\right)$, a within-group component $\left(b_{s}\right)$ and $\mathrm{F}_{\mathrm{ST}}$ calculated from $a_{s}$ and $b_{s}$ following Fumagalli [31] where:

and

$$
a_{s}=\frac{4 n_{i}\left(\widehat{p}_{(i, s)}-\widehat{p}_{s}\right)^{2}+4 n_{j}\left(\widehat{p}_{(j, s)}-\widehat{p}_{s}\right)^{2}-b_{s}}{2\left(2 n_{i} n_{j} /\left(n_{i}+n_{j}\right)\right)}
$$

where $\alpha_{(i, s)}=2 \widehat{p}_{(i, s)}\left(1-\widehat{p}_{(i, s)}\right)$ and $\alpha_{(j, s)}=2 \widehat{p}_{(j, s)}\left(1-\widehat{p}_{(j, s)}\right)$. $\widehat{p}_{(i, s)}$ is the coverage of a nucleotide at SNP site $(s)$ divided by the total coverage of $s$ in collection $(i) . n_{i}$ and $n_{j}$ are the number of mosquitoes sampled in collections $i$ and $j$, and $\widehat{p}_{s}$ is the coverage of a nucleotide at $s$ in both $i$ and $j$ collections divided by the total coverage of $s$ in both $i$ and $j$ collections. The estimate of $\mathrm{F}_{\mathrm{ST}}$ for $s$ is:

$$
F_{S T}(s)=\frac{a_{s}}{a_{s}+b_{s}}
$$

and for an entire gene $(g)$ with $m$ SNPs is:

$$
F_{S T}(g)=\frac{\sum_{s=1}^{m} a_{s}}{\sum_{s=1}^{m}\left(a_{s}+b_{s}\right)}
$$

Genes were annotated using Gene Ontology terms and SwissProt functional annotation data listed in AegyXcel (http://exon.niaid.nih.gov/transcriptome.html\#aegyxcel), using a cut-off e value of $\mathrm{E}^{-20}$.

Hardy-Weinberg expected heterozygosity $\left(H_{\text {exp }}\right)$ values were calculated for SNP sites that were present in both males and females using the following formula, $\alpha_{(i, s)}=2$ $\widehat{p}_{(i, s)}\left(1-\widehat{p}_{(i, s)}\right) ; \alpha_{(i, s)}$ is expected heterozygosity $\left(H_{\text {exp }}\right) \cdot \widehat{p}_{(i, s)}$ is the coverage of a variant at a $\operatorname{SNP}(s)$ site divided by the total coverage of $s$ in the collection $(i)$.

\section{Female-vs-female and male-vs-male comparisons}

Similar to the inter-sex comparisons, $\mathrm{F}_{\mathrm{ST}}$ was also calculated for replicate female SenAae and Aaa libraries (Pk10 female vs Thai female). $\mathrm{F}_{\mathrm{ST}}$ was also calculated for male-vs-male libraries to obtain the plots shown in Additional file 3 .

\section{Statistics}

Descriptive statistics were calculated in $\mathrm{R}$ (version 3.0.2). We evaluated $\mathrm{H}_{\exp }$ values along the length of chromosome 1 using a one-sided T-test $(p$ value $<0.05)$ that tested specifically for higher average $\mathrm{H}_{\exp }$ values in males over females. The ratio of variant sites per nucleotide of aligned reads was calculated as follows. Using flagstat (SAMtools), the number of aligned reads was determined in the reads aligned to reference *.bam files, and multiplied by the read-length (100nts) to achieve the total nucleotides aligned. The number of variants per chromosome was multiplied by 1000 and divided by the estimated total nucleotides aligned (Additional file 1).

Studies of Ae aegypti RAD-tag and SNP-CHIP analyses allowed just 2 alternate alleles per locus [73, 74], whereas, here, loci with 3 or more alternate alleles were included in the final analysis. Moreover, our approach is not subject to ascertainment bias, as occurs when a small number of SNPs from the entire genome are analyzed $[75,76]$. This systematic bias occurs when limited loci are analyzed rather than complete genotypic profiles.

\section{Additional files}

Additional file 1: Sequencing Statistics. SenAae (PK10) and Aaa (Thai) HTS details. Total trimmed reads aligned to the Aaeg $L 4$ reference; percent reads mapped; percent properly paired; number of variant sites; ratio of variant sites per aligned nucleotide*1000. (XLSX 16 kb)

Additional file 2: $F_{S T}$ frequency distributions. SenAae (PK10) and Aaa (Thai). (PDF $19 \mathrm{~kb}$ )

Additional file 3: $F_{S T}$ frequency distributions for female-vs-female and male-vs-male comparisons. SenAae (PK10) and Aaa (Thai). Black dots indicate average $F_{S T}$ values < 0.100; red dots indicate FST values $\geq 0.100$. (PDF $699 \mathrm{~kb}$ )

Additional file 4: Genes with significant sex-association values common to both populations. Vectorbase number (VBN), transcript, Chr, function, Function_description_Vectorbase. (XLSX 166 kb) 


\section{Abbreviations}

Aaa: Aedes aegypti aegypti; AaegL4: Chromosome-length Ae. aegypti aegypti reference sequence; blt: black tarsus locus; DNA: Deoxyribonucleic acid; $\mathrm{F}_{\mathrm{ST}}$ : Standardized variance, a measure of genetic association; $H_{\text {exp }}$ : Expected heterozygosity; $m$ allele: Female recessive locus; $M$ locus: Male determining locus; MD: Meiotic drive; PK10: Aedes aegypti collection from southeast Senegal; s: Spot locus; SenAae: Senegal Aedes aegypti collection; SNP: Singlenucleotide polymorphisms

\section{Acknowledgments}

We thank Karen Fleming for mosquito work and collections, as well as lab support. We thank Tyler Eike and Thomas Harrison for help with bioinformatics details. We also thank Mariangela Bonizzoni for helpful advice on the manuscript. The authors wish to thank the Franklin Graybill Statistical Laboratory at Colorado State University for statistical consulting.

\section{Funding}

This work was supported by National Institutes of Health (NIH) R01Al0833680 to WCB4. The funding body had no role in the study, collection, analysis, interpretation of data or in writing the manuscript.

\section{Availability of data and materials}

All Aaa (Thai) and SenAae (PK10) collection fastq sequences have been archived at the NCBI Sequence Read archive (SRA) under accession number PRJNA258086 (https://www.ncbi.nlm.nih.gov/sra/?term=PRJNA258086). Individual project numbers are as follows: Thai female replicate libraries: SRS682692 and SRS682687; Thai male replicates: SRX679429 and SRX679427. PK10 female replicates: SRX679424 and SRX679426; PK10 male replicates: SRX679423 and SRX679425. All scripts are available on request. Additional data is also available upon request.

\section{Authors' contributions}

CLC, LBD, and WCB4 designed the study. PJ and FJ provided the capture probe and gave technical advice on preparation details, probe design and capture probe details. CLC and LBD prepared the samples. SLF wrote scripts and provided guidance on data analysis. CLC and WCB4 analyzed the data. CLC and WCB4 wrote the manuscript. All authors read and approved the final manuscript.

\section{Ethics approval and consent to participate}

Not applicable, because this study does not involve humans, animals or plants. Field permissions were obtained for all collections.

\section{Consent for publication}

Not applicable

\section{Competing interests}

The authors declare that they have no competing interests.

\section{Publisher's Note}

Springer Nature remains neutral with regard to jurisdictional claims in published maps and institutional affiliations.

\section{Author details}

'Department of Microbiology, Immunology and Pathology, Colorado State University, Campus Delivery 1692, Fort Collins, CO 80523, USA. ²Department of Genetics, University of Cambridge, Downing Street, Cambridge CB2 3EH, UK.

Received: 15 June 2017 Accepted: 23 November 2017 Published online: 04 December 2017

\section{References}

1. Brown JE, McBride CS, Johnson P, Ritchie S, Paupy C, Bossin H, Lutomiah J, Fernandez-Salas I, Ponlawat A, Cornel AJ, Black WC IV, GorrochoteguiEscalante N, Urdaneta-Marquez L, Sylla M, Slotman M, Murray KO, Walker C, Powell JR. Worldwide patterns of genetic differentiation imply multiple 'domestications' of Aedes aegypti, a major vector of human diseases. Proc Biol Sci. 2011;278(1717):2446-54.
2. McClelland GAH. A worldwide survey of variation in scale pattern of the abdominal tergum of Aedea aegypti (L.) (Diptera:Culicidae). Trans Royal Entomol Soc Lond. 1974;126:239-59.

3. Tabachnick WJ, Powell JR. A world-wide survey of genetic variation in the yellow fever mosquito, Aedes aegypti. Genet Res. 1979;34(3):215-29.

4. Mattingly PF. Taxonomy of Aedes aegypti and related species. Bull World Health Organ. 1967;36(4):552-4.

5. Rasic G, Filipovic I, Weeks AR, Hoffmann AA. Genome-wide SNPs lead to strong signals of geographic structure and relatedness patterns in the major arbovirus vector, Aedes aegypti. BMC Genomics. 2014;15:275.

6. Dickson LB, Sanchez-Vargas I, Sylla M, Fleming K, Black WC IV. Vector competence in West African Aedes aegypti Is Flavivirus species and genotype dependent. PLoS Negl Trop Dis. 2014;8(10):e3153.

7. Sylla M, Bosio C, Urdaneta-Marquez L, Ndiaye M, Black WC IV. Gene flow, subspecies composition, and dengue virus-2 susceptibility among Aedes aegypti collections in Senegal. PLoS Negl Trop Dis. 2009;3(4):e408.

8. Presgraves DC, Orr HA. Haldane's rule in taxa lacking a hemizygous $X$ Science. 1998;282(5390):952-4.

9. Mattingly PF. Genetical aspects of the Aedes aegypti problem. I. Taxonom: and bionomics. Ann Trop Med Parasitol. 1957;51(4):392-408.

10. McClelland GA. Sex-linkage at two loci affecting eye pigment in the mosquito Aedes aegypti (diptera: culicidae). Can J Genet Cytol. 1966;8(2):192-8.

11. Newton ME, Southern DI, Wood RJ. X and Y chromosomes of Aedes aegypti (L.) distinguished by Giemsa C-banding. Chromosoma 1974, 49(1):41-49.

12. Hall AB, Basu S, Jiang X, Qi Y, Timoshevskiy VA, Biedler JK, Sharakhova MV, Elahi R, Anderson MA, Chen XG, Sharakhov IV, Adelman ZN, Tu Z. A male-determining factor in the mosquito Aedes aegypti. Science. 2015;348:1268.

13. Hall AB, Timoshevskiy VA, Sharakhova MV, Jiang X, Basu S, Anderson MA, Hu W, Sharakhov IV, Adelman ZN, Tu Z. Insights into the preservation of the homomorphic sex-determining chromosome of Aedes aegypti from the discovery of a male-biased gene tightly linked to the M-locus. Genome Biol Evol. 2014;6(1):179-91.

14. Salvemini M, D'Amato R, Petrella V, Aceto S, Nimmo D, Neira M, Alphey L, Polito LC, Saccone G. The orthologue of the fruitfly sex behaviour gene fruitless in the mosquito Aedes aegypti: evolution of genomic organisation and alternative splicing. PLoS One. 2013;8(2):e48554.

15. Salvemini M, Mauro U, Lombardo F, Milano A, Zazzaro V, Arca B, Polito LC, Saccone G. Genomic organization and splicing evolution of the doublesex gene, a drosophila regulator of sexual differentiation, in the dengue and yellow fever mosquito Aedes aegypti. BMC Evol Biol. 2011;11:41.

16. Clark NL, Aagaard JE, Swanson WJ. Evolution of reproductive proteins from animals and plants. Reproduction. 2006:131(1):11-22.

17. Coyne J, Orr HA. Speciation. Sunderland: Sinauer Associates; 2004

18. Swanson WJ, Vacquier VD. The rapid evolution of reproductive proteins. Nat Rev Genet. 2002;3(2):137-44.

19. Hosken DJ, Stockley P. Sexual selection and genital evolution. Trends Ecol Evol. 2004;19(2):87-93.

20. Krzywinska E, Kokoza V, Morris M, de la Casa-Esperon E, Raikhel AS, Krzywinski J. The sex locus is tightly linked to factors conferring sex-specific lethal effects in the mosquito Aedes aegypti. Heredity. 2016;117(6):408-16.

21. Krzywinska E, Krzywinski J. Analysis of expression in the Anopheles gambiae developing testes reveals rapidly evolving lineage-specific genes in mosquitoes. BMC Genomics. 2009;10:300.

22. Haerty W, Jagadeeshan S, Kulathinal RJ, Wong A, Ravi Ram K, Sirot LK, Levesque L, Artieri CG, Wolfner MF, Civetta A, Singh RS. Evolution in the fast lane: rapidly evolving sex-related genes in Drosophila. Genetics. 2007;177(3):1321-35.

23. CI W, Davis AW. Evolution of postmating reproductive isolation: the composite nature of Haldane's rule and its genetic bases. Am Nat. 1993;142(2):187-212.

24. Ellegren $\mathrm{H}$, Parsch J. The evolution of sex-biased genes and sex-biased gene expression. Nat Rev Genet. 2007:8(9):689-98.

25. Bachtrog D. Y-chromosome evolution: emerging insights into processes of Y-chromosome degeneration. Nat Rev Genet. 2013;14(2):113-24.

26. Dickson LB, Sharakhova MV, Timoshevskiy VA, Fleming KL, Caspary A, Sylla M, Black WC IV. Reproductive incompatibility involving Senegalese Aedes aegypti $(\mathrm{L})$ is associated with chromosome rearrangements. PLoS Negl Trop Dis. 2016;10(4):e0004626.

27. Dickson LB, Campbell CL, Juneja P, Jiggins FM, Sylla M, Black WC IV. Exon-enriched libraries reveal large genic differences between Aedes aegypti from Senegal, West Africa, and populations outside Africa. G3. $2017 ; 7(2): 571-82$ 
28. Fontaine A, Filipovic I, Fansiri T, Hoffmann AA, Cheng C, Kirkpatrick M, Rasic $G$, Lambrechts L. Extensive genetic differentiation between Homomorphic sex chromosomes in the mosquito vector, Aedes aegypti. Genome Biol Evol. 2017:9(9):2322-35.

29. Juneja P, Ariani CV, Ho YS, Akorli J, Palmer WJ, Pain A, Jiggins FM. Exome and Transcriptome sequencing of Aedes aegypti identifies a locus that confers resistance to Brugia malayi and alters the immune response. PLoS Pathog. 2015;11(3):e1004765.

30. Crawford JE, Alves JM, Palmer WJ, Day JP, Sylla M, Ramasamy R, Surendran SN, Black WC IV, Pain A, Jiggins FM. Population genomics reveals that an anthropophilic population of Aedes aegypti mosquitoes in West Africa recently gave rise to American and Asian populations of this major disease vector. BMC Biol. 2017;15(1):16

31. Fumagalli M, Vieira FG, Korneliussen TS, Linderoth T, Huerta-Sanchez E, Albrechtsen A, Nielsen R. Quantifying population genetic differentiation from next-generation sequencing data. Genetics. 2013;195(3):979-92.

32. Haldane JS. Sex ratio and unisexual sterility in hybrid animals. J Genetics. 1922;12:101-9.

33. Barreiro LB, Laval G, Quach H, Patin E, Quintana-Murci L. Natural selection has driven population differentiation in modern humans. Nat Genet. 2008;40(3):340-5.

34. VectorBase: a home for invertebrate vectors of human pathogens (http://www.vectorbase.org/).

35. Dudchenko O, Batra SS, Omer AD, Nyquist SK, Hoeger M, Durand NC, Shamim MS, Machol I, Lander ES, Aiden AP, Aiden EL. De novo assembly of the Aedes aegypti genome using $\mathrm{Hi}-\mathrm{C}$ yields chromosome-length scaffolds. Science. 2017;356(6333):92-5.

36. Nene V, Wortman JR, Lawson D, Haas B, Kodira C, ZJ T, Loftus B, Xi Z Megy K, Grabherr M, Ren Q, Zdobnov EM, Lobo NF, Campbell KS, Brown SE, Bonaldo MF, Zhu J, Sinkins SP, Hogenkamp DG, Amedo P, Arsenburger P, Atkinson PW, Bidwell S, Biedler J, Birney E, Bruggner RV, Costas J, Coy MR, Crabtree J, Crawford M, Debruyn B, Decaprio D, Eiglmeier K, Eisenstadt E, El-Dorry H, Gelbart WM, Gomes SL, Hammond M, Hannick LI, Hogan JR, Holmes MH, Jaffe D, Johnston SJ, Kennedy RC, Koo H, Kravitz S, Kriventseva EV, Kulp D, Labutti K, Lee E, Li S, Lovin DD, Mao C, Mauceli E, Menck CF, Miller JR, Montgomery P, Mori A, Nascimento AL, Naveira HF, Nusbaum C, O'Leary SB, Orvis J, Pertea M, Quesneville H, Reidenbach KR, Rogers YH, Roth CW, Schneider JR, Schatz M, Shumway M, Stanke M, Stinson EO, Tubio JM, Vanzee JP, Verjovski-Almeida S, Werner D, White O, Wyder S, Zeng Q, Zhao Q, Zhao Y, Hill CA, Raikhel AS, Soares MB, Knudson DL, Lee NH, Galagan J, Salzberg SL, Paulsen IT, Dimopoulos G, Collins FH, Bruce B, FraserLiggett CM, Severson DW. Genome sequence of Aedes aegypti, a major Arbovirus vector. Science. 2007;316:1718.

37. Hickey WA, Craig GB Jr. Distortion of sex ratio in populations of Aedes aegypti. Can J Genet Cytol. 1966;8(2):260-78

38. Hoang KP, Teo TM, Ho TX, Le VS. Mechanisms of sex determination and transmission ratio distortion in Aedes aegypti. Parasit Vectors. 2016;9:49.

39. Wood RJ, Newton ME. Sex-ratio distortion caused by meiotic drive in mosquitoes. Am Nat. 1991;137(3):379-91.

40. Lucotte EA, Laurent R, Heyer E, Segurel L, Toupance B. Detection of allelic frequency differences between the sexes in humans: a signature of sexually antagonistic selection. Genome Biol Evol. 2016;8(5):1489-500.

41. Cheng C, Kirkpatrick M. Sex-specific selection and sex-biased gene expression in humans and flies. PLoS Genet. 2016;12(9):e1006170.

42. Coolon JD, Stevenson KR, McManus CJ, Yang B, Graveley BR, Wittkopp PJ. Molecular mechanisms and evolutionary processes contributing to accelerated divergence of gene expression on the drosophila $X$ chromosome. Mol Biol Evol. 2015;32(10):2605-15.

43. AegyXcel; http://exon.niaid.nih.gov/transcriptome.html\#aegyxcel.

44. McBride CS, Baier F, Omondi AB, Spitzer SA, Lutomiah J, Sang R, Ignell R, Vosshall LB. Evolution of mosquito preference for humans linked to an odorant receptor. Nature. 2014;515(7526):222-7.

45. Sparks JT, Bohbot JD, Dickens JC. The genetics of chemoreception in the labella and tarsi of Aedes aegypti. Insect Biochem Mol Biol. 2014;48:8-16

46. Dubendorfer A, Hediger M, Burghardt G, Bopp D. Musca domestica, a window on the evolution of sex-determining mechanisms in insects. Int J Dev Biol. 2002:46(1):75-9.

47. Franco MG, Rubini PG, Vecchi M. Sex-determinants and their distribution in various populations of Musca domestica L. of Western Europe. Genet Res. 1982;40(3):279-93.
48. Thompson PE, Bowen JS. Interactions of differentiated primary sex factors in Chironomus Tentans. Genetics. 1972;70(3):491-3.

49. Black DL. Mechanisms of alternative pre-messenger RNA splicing. Annu Rev Biochem. 2003;72:291-336.

50. Marinho J, Martins T, Neto M, Casares F, Pereira PS. The nucleolar protein Viriato/Nol12 is required for the growth and differentiation progression activities of the Dpp pathway during Drosophila eye development. Dev Biol. 2013;377(1):154-65.

51. Sirot LK, Hardstone MC, Helinski ME, Ribeiro JM, Kimura M, Deewatthanawong P, Wolfner MF, Harrington LC. Towards a semen proteome of the dengue vector mosquito: protein identification and potential functions. PLoS Negl Trop Dis. 2011;5(3):e989.

52. Charlesworth B. The evolution of chromosomal sex determination and dosage compensation. Curr Biol. 1996;6(2):149-62.

53. Lahn BT, Pearson NM, Jegalian K. The human Y chromosome, in the light of evolution. Nat Rev Genet. 2001;2(3):207-16.

54. Lemaitre C, Braga MD, Gautier C, Sagot MF, Tannier E, Marais GA. Footprints of inversions at present and past pseudoautosomal boundaries in human sex chromosomes. Genome Biol Evol. 2009;1:56-66.

55. Graham DH, Holmes JL, Black WC IV. Identification of quantitative trait loci affecting sex determination in the eastern treehole mosquito (Ochlerotatus triseriatus). J Hered. 2004;95(1):35-45.

56. Christophers S. Aedes aegypti (L.), the yellow-fever mosquito; its life history, bionomics and structure. London: Cambridge University Press; 1960.

57. Craig GB Jr, Vandehey RC, Hickey WA. Genetic variability in populations of Aedes aegypti. Bull World Health Organ. 1961;24:527-39.

58. Newton ME, Wood RJ, Southern DI. A cytogenetic analysis of meiotic drive in the mosquito, Aedes aegypti (L.). Genetica. 1976;46(3):297-318.

59. Hickey WA, Craig GB Jr. Genetic distortion of sex ratio in a mosquito, Aedes aegypti. Genetics. 1966;53(6):1177-96.

60. Owusu-Daaku KO, Wood RJ, Butler RD. Selected lines of Aedes aegypti with persistently distorted sex ratios. Heredity. 1997;79(Pt 4):388-93.

61. Suguna SG, Wood RJ, Curtis CF, Whitelaw A, Kazmi SJ. Resistance to meiotic drive at the MD locus in an Indian wild population of Aedes aegypti. Genet Res. 1977;29(2):123-32.

62. Wood RJ. Between-family variation in sex ratio in the Trinidad (T-30) strain of Aedes aegypti (L.) indicating differences in sensitivity to the meiotic drive gene MD. Genetica. 1976;46(3):345-61.

63. Mori A, Chadee DD, Graham DH, Severson DW. Reinvestigation of an endogenous meiotic drive system in the mosquito, Aedes aegypti (Diptera: Culicidae). J Med Entomol. 2004;41(6):1027-33.

64. Craig GB Jr, Hickey WA, Vandehey RC. An inherited male-producing factor in Aedes aegypti. Science. 1960;132(3443):1887-9.

65. Wood RJ, Ouda NA. The genetic basis of resistance and sensitivity to the meiotic drive gene D in the mosquito Aedes aegypti L. Genetica. 1987;72(1):69-79.

66. Bosio CF, Harrington LC, Jones JW, Sithiprasasna R, Norris DE, Scott TW. Genetic structure of Aedes aegypti populations in Thailand using mitochondrial DNA. Am J Trop Med Hyg. 2005;72(4):434-42.

67. Huber K, Ba Y, Dia I, Mathiot C, Sall AA, Diallo M. Aedes aegypti in Senegal: genetic diversity and genetic structure of domestic and sylvatic populations. Am J Trop Med Hyg. 2008;79(2):218-29.

68. Martin M. Cutadapt removes adapter sequences from high-throughput sequencing reads. EMBnet J. 2011;17(1):10-2.

69. TD W, Nacu S. Fast and SNP-tolerant detection of complex variants and splicing in short reads. Bioinformatics. 2010;26(7):873-81.

70. Li H, Handsaker B, Wysoker A, Fennell T, Ruan J, Homer N, Marth G, Abecasis G, Durbin R, Genome Project Data Processing S. The sequence alignment/ map format and SAMtools. Bioinformatics. 2009;25(16):2078-9.

71. Koboldt DC, Zhang Q, Larson DE, Shen D, McLellan MD, Lin L, Miller CA, Mardis ER, Ding L, Wilson RK. VarScan 2: somatic mutation and copy number alteration discovery in cancer by exome sequencing. Genome Res. 2012;22(3):568-76.

72. Ebbert MT, Wadsworth ME, Staley LA, Hoyt KL, Pickett B, Miller J, Duce J, Alzheimer's Disease Neuroimaging I, Kauwe JS, Ridge PG. Evaluating the necessity of PCR duplicate removal from next-generation sequencing data and a comparison of approaches. BMC Bioinf. 2016;17(Suppl 7):239.

73. Brown JE, Evans BR, Zheng W, Obas V, Barrera-Martinez L, Egizi A, Zhao H, Caccone A, Powell JR. Human impacts have shaped historical and recent evolution in Aedes aegypti, the dengue and yellow fever mosquito. Evolution. 2014;68(2):514-25. 
74. Evans BR, Gloria-Soria A, Hou L, McBride C, Bonizzoni M, Zhao H, Powell JR. A multipurpose, high-throughput single-nucleotide polymorphism chip for the dengue and yellow fever mosquito, Aedes aegypti. G3. 2015;5(5):711-8.

75. Frascaroli E, Schrag TA, Melchinger AE. Genetic diversity analysis of elite European maize (Zea mays L.) inbred lines using AFLP, SSR, and SNP markers reveals ascertainment bias for a subset of SNPS. Theor Appl Genet. 2013;126(1):133-41.

76. Seeb JE, Carvalho G, Hauser L, Naish K, Roberts S, Seeb LW. Singlenucleotide polymorphism (SNP) discovery and applications of SNP genotyping in nonmodel organisms. Mol Ecol Resour. 2011;11(Suppl 1):1-8.

Submit your next manuscript to BioMed Central and we will help you at every step:

- We accept pre-submission inquiries

- Our selector tool helps you to find the most relevant journal

- We provide round the clock customer support

- Convenient online submission

- Thorough peer review

- Inclusion in PubMed and all major indexing services

- Maximum visibility for your research

Submit your manuscript at www.biomedcentral.com/submit
Biomed Central 